\title{
Spherical Growth and Surface-Quasifree Vibrations of Si Nanocrystallites in Er-Doped Si Nanostructures
}

\author{
X. L. Wu, ${ }^{1}$ Y. F. Mei, ${ }^{1}$ G. G. Siu, ${ }^{2}$ K. L. Wong, ${ }^{3}$ K. Moulding, ${ }^{3}$ M. J. Stokes, ${ }^{2}$ C. L. Fu, ${ }^{2}$ and X. M. Bao ${ }^{1}$ \\ ${ }^{1}$ National Laboratory of Solid State Microstructures and Department of Physics, Nanjing University, \\ Nanjing 210093, People's Republic of China \\ ${ }^{2}$ Department of Physics and Materials Science, City University of Hong Kong, Kowloon, Hong Kong, People’s Republic of China \\ ${ }^{3}$ Materials Characterization and Preparation Facility, Hong Kong University of Science and Technology, \\ Kowloon, Hong Kong, People's Republic of China
}

(Received 28 January 2000; revised manuscript received 28 November 2000)

\begin{abstract}
Si-based Er-doped Si nanostructures were fabricated for exploring efficient light emission from Er ions and $\mathrm{Si}$ nanocrystallites. High-resolution transmission electron microscopy observations reveal that $\mathrm{Si}$ nanocrystallites are spherically embedded in the $\mathrm{SiO}_{2}$ matrix. Energy-dispersive x-ray analysis indicates that the Er centers are distributed at the surfaces of nanocrystallites surrounded by the $\mathrm{SiO}_{2}$ matrix. Lowfrequency Raman scattering investigation shows that Lamb's theory can be adopted to exactly calculate the surface vibration frequencies from acoustic phonons confined in spherical Si nanocrystallites and the matrix effects are negligible.
\end{abstract}

PACS numbers: $61.46 .+\mathrm{w}$

Er ions have played an important role in developing optical communication technology [1]. Si nanostructures have also shown large application potentials in optoelectronics [2-7]. Thus, it is very significant to explore efficient light-emitting materials in which electrical and optical functions from $\mathrm{Er}$ ions and $\mathrm{Si}$ nanocrystallites may be realized on a single Si chip. Radio-frequency magnetron sputtering has been proved to be a useful technique for fabricating this kind of $\mathrm{Si}$ nanostructures [7,8]. To further obtain efficient light emission from $\mathrm{Er}$ ions at $1.54 \mu \mathrm{m}$, we may introduce Er ions into the materials. Since the bonding nature of the $\mathrm{SiO}_{2}$ network could provide the environment for the $\mathrm{Er}$ to be incorporated into an active configuration, the material fabrications should be carried out under an adequate oxygen pressure [1].

In this Letter, we report fabrications of Si-based Er-doped Si nanostructures by magnetron cosputtering from metallic $\mathrm{Er}$ and $\mathrm{Si}$ targets in diluted oxygen. We mainly reveal that the presence of Er centers makes it possible to perfectly grow spherical $\mathrm{Si}$ nanocrystallites in the $\mathrm{SiO}_{2}$ matrix, which will be used to clarify many physical problems in controversy related to the size, shape, and confined property of Si nanocrystallites [7-9]. Here we investigate low-frequency Raman scattering from the surface vibrations of spherical Si nanocrystallites. Good agreement is achieved between the observed Raman peaks and theoretical prediction.

The sample depositions were conducted in a conventional magnetron sputtering chamber evacuated to a base pressure of $<10^{-6}$ Torr. The substrates were $\langle 100\rangle$ oriented $p$-type $\mathrm{Si}$ wafers with $1-3 \Omega \mathrm{cm}$ resistivity. The samples were obtained by cosputtering a silicon target covered with metallic Er platelets under an oxygen pressure of $5 \times 10^{-5}$ Torr and the power of $120 \mathrm{~W}$. The substrate temperatures were held at $350{ }^{\circ} \mathrm{C}$. Rapid thermal annealing was performed in a controlled $\mathrm{N}_{2}$ atmosphere at temperatures varying from 600 to $1100{ }^{\circ} \mathrm{C}$ for $2 \mathrm{~min}$ to form $\mathrm{Si}$ nanocrystallites by $\mathrm{Si}$ segregation and $\mathrm{Er}$ redistribution at the moving phase boundary $[10,11]$. The film thickness was determined by means of angle lapping to be about $1.8 \mu \mathrm{m}$. Infrared absorption measurements indicated that the $\mathrm{Si}$ oxide is almost stoichiometric $\mathrm{SiO}_{2}$. The Er concentration was estimated to be $\sim 3$ at. \% in as-sputtered samples. The films were mainly characterized using high-resolution transmission electron microscopy (HRTEM, Phillips CM200 FEG TEM, equipped with energy-dispersive detection of the $\mathrm{x}$ rays emitted by an area of about $\pi \times 2^{2} \mathrm{~nm}^{2}$ of the sample), visible photoluminescence (PL, Hitachi 850 equipped with infrared detector), and Raman spectroscopy (SPEX 1403 RAMALOG system). Both polarized and depolarized Raman spectra were obtained at back-scattering geometry using two excitation lines (488 and $514.5 \mathrm{~nm}$ ) of $\mathrm{Ar}^{+}$laser [12]. The power illuminating on the sample was $16 \mathrm{~mW}$ focused to an area of about $\pi \times 0.8^{2} \mathrm{~mm}^{2}$. Under our experimental conditions, all measured spectra were corrected for the polarization-dependent response of the measurement system. During Raman measurements, the samples were kept in a vacuum to avoid the signal from air.

For all fabricated samples, two sharp PL peaks in the infrared can be observed at 1.535 and $1.550 \mu \mathrm{m}$, as shown in Fig. 1(a). Their positions remain unchanged, but their intensities vary with the annealing temperature. Since the $\mathrm{Er}^{3+}$-related emission energy is rather independent of its environment and temperature [1], the two sharp infrared PL peaks should be a result of the ${ }^{4} I_{13 / 2} \rightarrow{ }^{4} I_{15 / 2}$ transitions in $\mathrm{Er}^{3+}$. The PL intensity variation is a signature for change of the $\mathrm{Er}^{3+}$ bonding environment. Shown in Fig. 1(b) are the corresponding visible PL spectra. The peak positions are all at about $620 \mathrm{~nm}$ and no crystallite size dependence is observed, but their intensities change with annealing temperature, To understand the origin of 

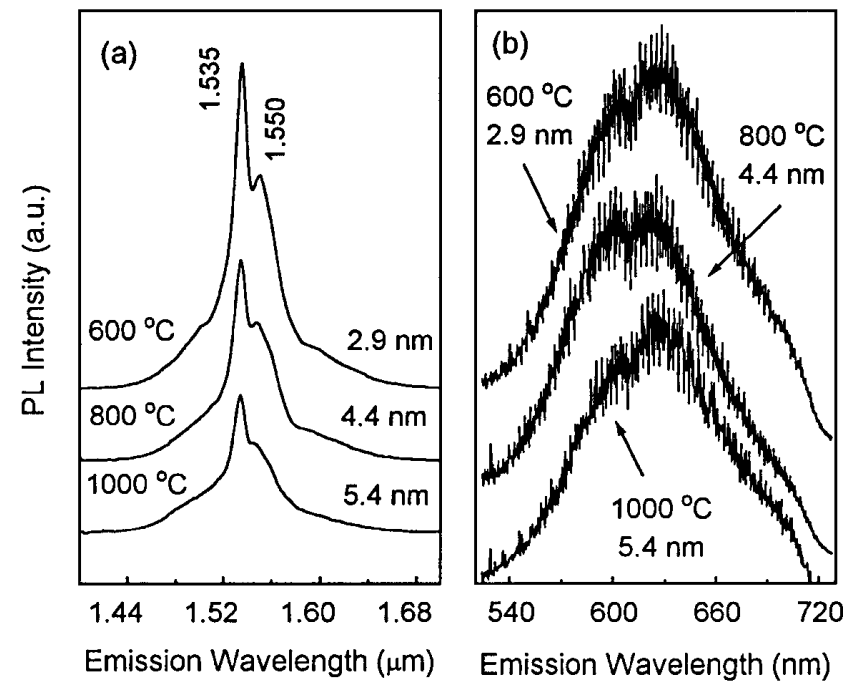

FIG. 1. (a) Infrared and (b) visible PL spectra from three Er-doped samples annealed at 600,800 , and $1000{ }^{\circ} \mathrm{C}$.

the visible PL, the PL excitation spectra were examined and an excitation peak at about $400 \mathrm{~nm}$ was observed in all samples. This result indicates that the visible PL has an origin related to the Si nanocrystallites [13]. Based on previous results $[3,13-15]$, we suggest that the visible PL arises from optical transitions in the $\mathrm{Si}=\mathrm{O}$ binding states at the surfaces of Si nanocrystallites [4,15-17].

To check the existence of $\mathrm{Si}$ nanocrystallites, planar and cross-section TEM observations were carried out on all samples. Three typical TEM images from the samples annealed at 600,800 , and $1100{ }^{\circ} \mathrm{C}$ are presented in Figs. 2(a)-2(c), respectively. These photographs clearly display the presence of spherical Si nanocrystallites (dark patches), and they are well dispersed in the $\mathrm{SiO}_{2}$ matrix. A HRTEM image of the crystallites from the sample annealed at $800{ }^{\circ} \mathrm{C}$ is shown in Fig. 2(d). We can clearly see that the large Si crystallite is perfectly spherical and has lattice fringes corresponding to the $\{111\}$ plane of Si. This kind of perfectly spherical growth can be observed only in our samples with Er doping and cannot take place in the samples without Er cosputtering [8]. According to the obtained TEM results, the mean sizes $(d)$ and size distributions (standard deviation $\sigma$ ) of Si nanocrystallites can be characterized by a log-normal function [18]. For the samples annealed at 600,800 , and $1000{ }^{\circ} \mathrm{C}, d=2.9$, 4.4 , and $5.4 \mathrm{~nm}$ and $\sigma=1.01,1.88$, and $2.82 \mathrm{~nm}$, respectively. In all samples, we found that $d$ and $\sigma$ increase with annealing temperature. In the sample annealed at $1100{ }^{\circ} \mathrm{C}, d$ and $\sigma$ abruptly increase to $\sim 9.8$ and $6.18 \mathrm{~nm}$, respectively, but no micropin holes were observed in the $\mathrm{SiO}_{2}$ network [19]. This indicates that the Er existence is beneficial to growth of $\mathrm{Si}$ crystallites and formation of dense $\mathrm{SiO}_{2}$.

To identify the positions of $\mathrm{Er}$ centers, energydispersive $\mathrm{x}$-ray fluorescence (EDXF) measurements were performed at different positions around nanocrystallites. Figures 3(a)-3(d) show the corresponding results from

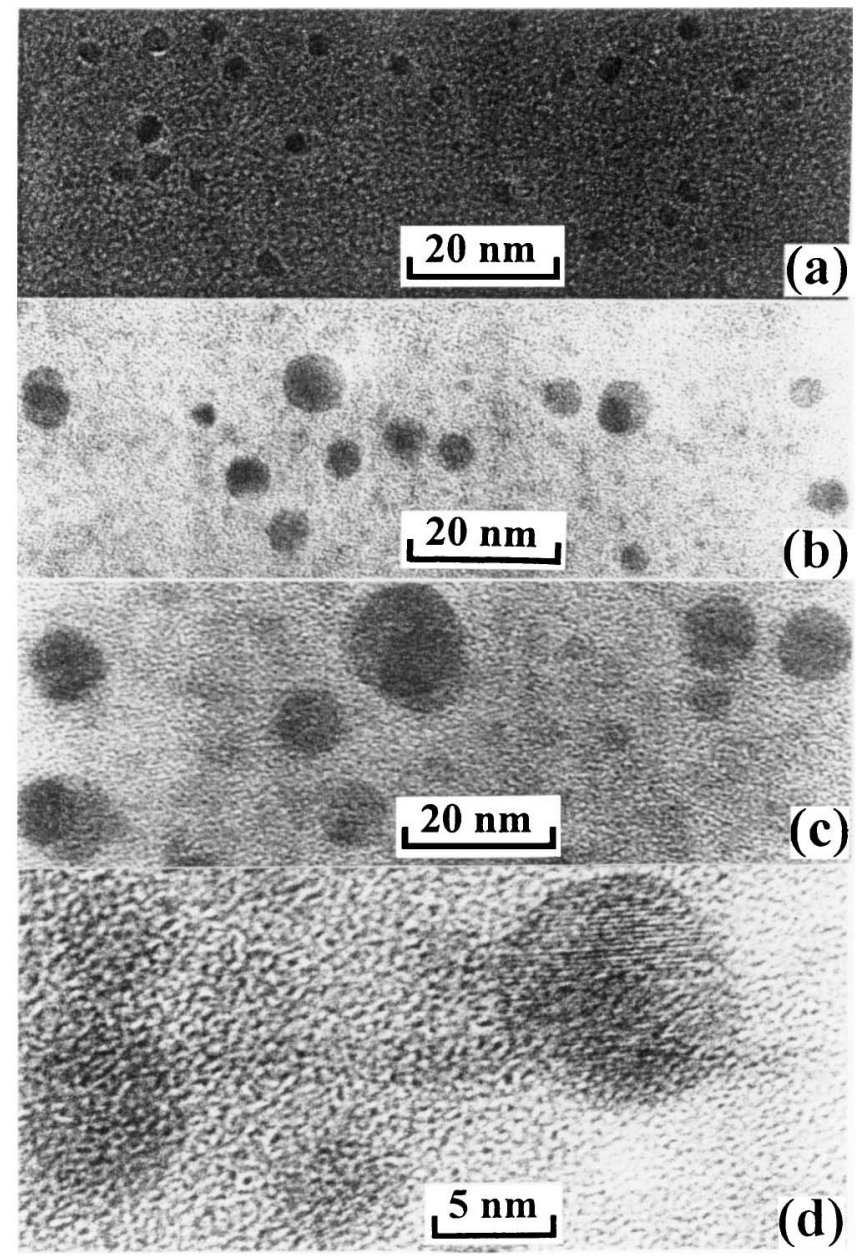

FIG. 2. TEM images of three typical samples annealed at (a) $600{ }^{\circ} \mathrm{C}$, (b) $800{ }^{\circ} \mathrm{C}$, and (c) $1100{ }^{\circ} \mathrm{C}$. (d) A HRTEM image of the crystallites. Spherical Si crystallites embedded in the $\mathrm{SiO}_{2}$ matrix can clearly be seen with lattice fringes corresponding to the $\{111\}$ plane of Si.

some typical positions. At the edges of crystallites, Er peak exists only at some positions. Since electronic beam has more overlapping with the crystallite, the $\mathrm{Si}$ peak shows larger intensity in spectrum 3(b) than in spectrum 3(a). In the matrix and inside crystallite, no Er characteristic peak is observed, indicating that the Er centers mainly distribute at some positions of the surfaces of Si crystallites. They do not form an Er shell around $\mathrm{Si}$ crystallite. These Er centers may reduce defects around $\mathrm{Si}$ crystallites and thus lead to perfectly spherical growth of Si crystallites. Further x-ray absorption fine structure measurements show that $\mathrm{Er}$ is coordinated with six oxygen atoms in a first shell. So we conclude that the $\mathrm{Er}$ is not directly bonded to Si but connected through a bridging oxygen atom. This is the reason for having strong $1.54 \mu \mathrm{m}$ PL in our samples.

Below, we present an investigation on the surface vibration properties of spherical Si nanocrystallites by examining low-frequency Raman spectra. Recently, vibration properties of small solid particles have become a current subject of intense controversy [8,9,20-23]. A key issue 


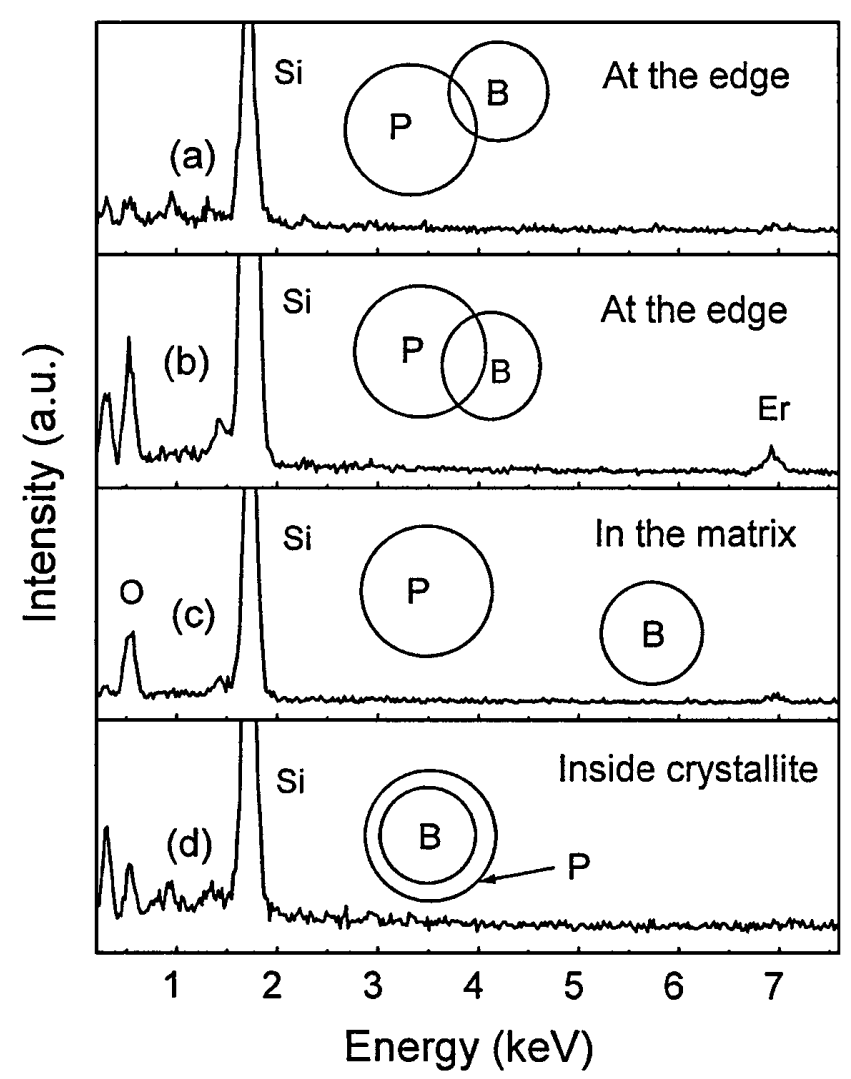

FIG. 3. Some typical EDXF spectra emitted at different positions around Si crystallites: (a) and (b) at the edges of Si crystallite; (c) in the matrix; (d) inside Si crystallite. $P$ : Si crystallite; $B$ : electronic beam focused at an area of about $\pi \times 2^{2} \mathrm{~nm}^{2}$.

is whether Lamb's theory [24] about the vibrations of a small elastic body with a free boundary condition could be applied to a small particle embedded in a matrix. Different research groups even presented completely opposite predictions for influence of the matrix. Fujii et al. [8] reported, for the first time, the low-frequency Raman scattering results of $\mathrm{Si}$ crystallites embedded in the $\mathrm{SiO}_{2}$, but the calculated frequencies from the acoustic phonons confined in Si crystallites are much larger than those of the observed peaks. An obvious fact in their samples is that the shapes of Si nanocrystallites completely deviate from sphericity [8].

Figure 4(a) shows four polarized low-frequency Raman spectra from our Er-doped samples. These Raman peaks have the following features: (1) The Raman shifts decrease with increasing the Si crystallite size. The peak frequencies are almost inversely proportional to the crystallite diameters, as shown in Fig. 5. For each Raman peak, its frequency remains unchanged with the excitation line [see Fig. 4(b)]; (2) If the volume-mean diameter $d_{\nu}$ of Si crystallites is calculated using the log-normal function, we can find that the samples annealed at 600, 800, 1000, and $1100{ }^{\circ} \mathrm{C}$ have $d_{\nu}=3.1,4.8,5.9$, and $10.8 \mathrm{~nm}$, respectively. Obviously, the sample with the largest $d_{\nu}$ displays the strongest Raman peak [25]; (3) The depolarized spectra [see Fig. 4(c)] are similar to those observed for CdS
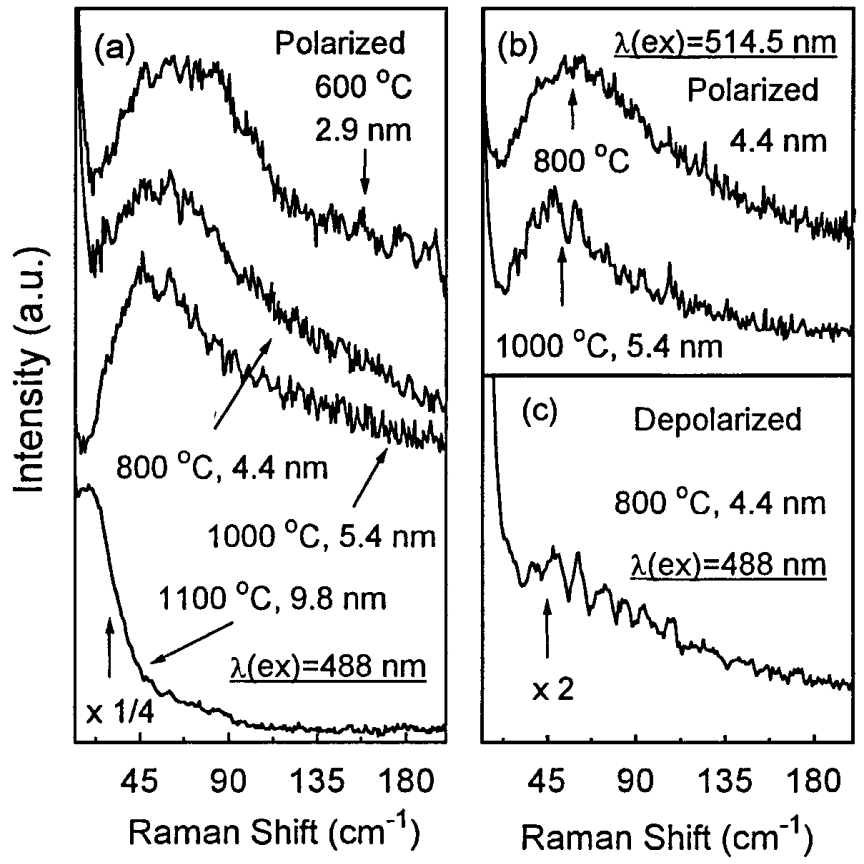

FIG. 4. Polarized (a) and (b) and depolarized (c) lowfrequency Raman spectra of some samples, measured with two excitation lines of 488 and $514.5 \mathrm{~nm}$ of $\mathrm{Ar}^{+}$laser.

nanocrystallites embedded in $\mathrm{a} e O_{2}$ glass matrix [22]. No depolarization property was observed. The above results suggest that these Raman peaks are related to scattering from the confined acoustic phonons which arise from the elastic vibrations of spherical Si nanocrystallites.

According to the usual vibration theory of elastic body presented by Lamb, two types of confined acoustic phonon (spheroidal and torsional) modes can be obtained in a spherical crystallite. Their eigenfrequencies are characterized by two quantum numbers of $l$ and $n$. The spheroidal mode of $l=0$ is purely radial with spherical symmetry. It produces totally polarized spectra and thus can easily be observed in the low-frequency Raman spectra according to the section rules [26]. Currently, these Raman peaks should be from the contribution of a spheroidal mode with $l=0$ and $n=0$. To theoretically calculate the eigenfrequencies of the confined acoustic modes from an elastic sphere with free surface, we must take into account the anisotropy of sound propagation in Si. Sound velocities were derived from elastic constants $c_{11}=16.57, c_{12}=$ 6.39 , and $c_{44}=7.96 \times 10^{10} \mathrm{~N} / \mathrm{m}^{2}$, and a mass density $\rho=2332 \mathrm{~kg} / \mathrm{m}^{3}$ [8]. We use sound velocities propagating in $\langle 100\rangle,\langle 111\rangle$, and $\langle 110\rangle$ directions to obtain the Raman peak frequencies of the spheroidal modes with $l=0$ [20]. The corresponding results are presented in Fig. 5. Obviously, excellent agreement is achieved between experiments and calculations, indicating that for spherical nanocrystallites Lamb's theory with the free boundary condition can be used to exactly calculate the vibration frequencies of a crystallite embedded in a matrix.

Are the positions of the Raman peaks the influence of $\mathrm{Er}$ oxide at the surface of Si crystallite? We can identify the 


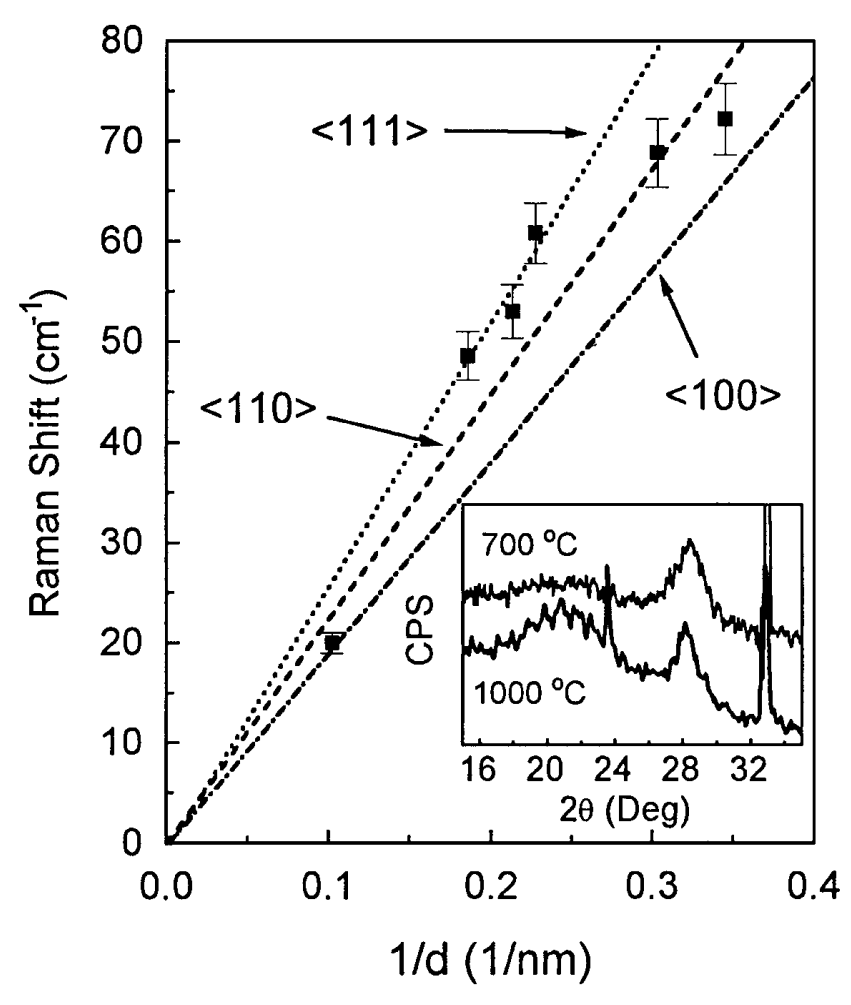

FIG. 5. Dependence of the polarized Raman peak frequencies (filled squares) on the inverse Si nanocrystallite diameters. Dotted, broken, and dash-dotted lines are the results of theoretical calculations for the confined acoustic modes (spherical modes with $l=0$ ) assuming the sound velocities in $\langle 111\rangle,\langle 110\rangle$, and $\langle 100\rangle$ directions, respectively. The inset is two $\mathrm{x}$-ray diffraction spectra from the samples annealed at 700 and $1000^{\circ} \mathrm{C}$.

influence by calculating the compressive stress exerted on Si crystallite $[9,27]$. The inset of Fig. 5 shows two typical $\mathrm{x}$-ray diffraction spectra. From the shift of the diffraction angle at about $2 \theta=28^{\circ}$ to the position of single crystal, we can obtain the stress $s$ to be less than $0.5 \%$ in all samples. Using the formula $\Delta \omega=-s(P+2 Q) / 2 \omega_{c}$, where $P=-1.43 \omega_{c}^{2}$ and $Q=-1.89 \omega_{c}^{2}$ are the phonon deformation potentials of $\mathrm{Si}$, and $\omega_{c}$ is the frequency of the confined acoustic phonon (in stress-free case), we can roughly estimate the magnitude of the Raman redshift $\Delta \omega$ induced by the stress to be less than $2 \mathrm{~cm}^{-1}$. Hence, this influence is negligible [21,22]. In the literature [28], some sharp low-frequency Raman peaks were observed for quasi-free $\mathrm{Si}$ clusters embedded in porous $\mathrm{Si}$ and attributed to the combination of different modes with TA phonons. However, why does not a similar phenomenon appear in our present results? This can be explained as follows: (1) Second-order Raman scattering by two phonons of surface and TA modes is a surface-assisted process. A different surface condition of Si clusters can cause a different combination of two phonons [28]. Therefore, the existence of both the Er centers and the $\mathrm{SiO}_{2}$ matrix at the surfaces of crystallites possibly eliminates this combination and thus only causes a first-order surface mode; (2) the multimode feature is connected with cluster shape deformation [28].
Spherical nanocrystallites may cause only the first-order feature of the surface mode.

In summary, we have fabricated Si-based Er-doped $\mathrm{Si}$ nanostructures with light emission properties from both Er centers and Si nanocrystallites. Spherical growth of $\mathrm{Si}$ nanocrystallites are observed to be due to the existence of Er centers at the surfaces of Si crystallites. Low-frequency Raman results indicate that the vibration property of small particles embedded in a matrix mainly depends on their shapes and the matrix effect is negligible.

This work was supported by Grants No. 59832100 and No. 60076007 from the National Natural Science Foundation of China.

[1] For example, see a review report, A. Polman, J. Appl. Phys. 82, 1 (1997).

[2] S. S. Lyer and Y.H. Xie, Science 260, 40 (1993).

[3] Y. Kanemitsu, Phys. Rev. B 48, 4883 (1993); Y. Kanemitsu, H. Uto, and Y. Masumoto, ibid. 48, 2827 (1993).

[4] S. M. Prokes and O. J. Glembocki, Phys. Rev. B 49, 2238 (1994); S. M. Prokes, W.E. Carlos, and O. J. Glembocki, ibid. 50, 17093 (1994).

[5] S. Veprek et al., Appl. Phys. Lett. 67, 2215 (1995).

[6] K. D. Hirschman, L. Tsybeskov, S. P. Duttagupta, and P. M. Fauchet, Nature (London) 384, 338 (1996).

[7] C. A. Gullis, L. T. Canham, and P.D. J. Calcott, J. Appl. Phys. 82, 909 (1997).

[8] M. Fujii, Y. Kanzawa, S. Hayashi, and K. Yamamoto, Phys. Rev. B 54, R8373 (1996).

[9] N. N. Ovsyuk and V. N. Novikov, Phys. Rev. B 53, 3113 (1996).

[10] J. A. Capobianco, P. P. Proulx, B. Andrianasolo, and B. Champagnon, Phys. Rev. B 43, 10031 (1991).

[11] A. Polman et al., J. Appl. Phys. 81, 150 (1997).

[12] X. L. Wu et al., Phys. Rev. B 57, 9945 (1998).

[13] Y.H. Xie et al., J. Appl. Phys. 71, 2403 (1992).

[14] K. Koch and V. Petrova-koch, Porous Silicon, edited by Z. C. Feng and R. Tsu (World Scientific, Singapore, 1994), p. 133.

[15] M. V. Wolkin et al., Phys. Rev. Lett. 82, 197 (1999).

[16] J. L. Gole, F. P. Dudel, D. Grantier, and D. A. Dixon, Phys. Rev. B 56, 2137 (1997).

[17] X. L. Wu et al., Phys. Rev. B 62, R7759 (2000).

[18] K. Tsunetomo et al., Jpn. J. Appl. Phys. 29, 2481 (1990).

[19] X. L. Wu et al., Appl. Phys. Lett. 74, 2420 (1999).

[20] L. Saviot et al., J. Non-Cryst. Solids 197, 238 (1996).

[21] M. Fujii, T. Nagareda, and K. Yamamoto, Phys. Rev. B 44, 6243 (1991).

[22] A. Tanaka, S. Onari, and T. Arai, Phys. Rev. B 47, 1237 (1993).

[23] A. Tamura, K. Higeta, and T. Ichinokawa, J. Phys. C 15, 4975 (1985).

[24] H. Lamb, Proc. London Math. Soc. 13, 187 (1882).

[25] M. Montagna and R. Dusi, Phys. Rev. B 52, 10080 (1995).

[26] E. Duval, Phys. Rev. B 46, 5795 (1992).

[27] H. Xia et al., J. Appl. Phys. 78, 6705 (1995); X.L. Wu et al., ibid. 82, 2704 (1997); X. L. Wu et al., Appl. Phys. Lett. 70, 838 (1997).

[28] F. Q. Liu et al., Phys. Rev. Lett. 76, 604 (1996). 